\title{
Categorias evolutivas para radiologistas conquistarem a perícia
}

\author{
Patrícia Fernanda Dorow Doutora em Engenharia e Gestão do Conhecimento. Instituto Federal de Santa Catarina (IFSC) - \\ Brasil. patriciad@ifsc.edu.br \\ Gregório Varvakis Doutor em Manufacturing Engineering. Universidade Federal de Santa Catarina (UFSC) - Brasil. \\ gvarvaki@ufsc.br \\ José Cláudio Terra Doutor em Engenharia de Produção. Hospital Albert Einstein - Brasil. terra@gmail.com \\ Luiz Felipe de Souza Nobre Doutor em Medicina. Universidade Federal de Santa Catarina (UFSC) - Brasil. nobre@gmail.com
}

\section{RESUMO}

O objetivo do artigo é identificar e descrever as categorias evolutivas para um radiologista conquistar a perícia. O estudo é respaldado na teoria de aprendizagem de Bloom et al. (1956), que permite verificar os resultados de aprendizagem. A partir de um estudo qualitativo, é apresentado o processo de aprendizagem de radiologistas novatos e especialistas e explicitado cada passo necessário para se tornar um especialista em um domínio do diagnóstico por imagem. Participaram do estudo 43 de três organizações de radiologia e diagnóstico por imagem, sendo 21 especialistas e 28 novatos. A coleta dos dados foi realizada por meio de observações, entrevistas semiestruturadas e verificação dos resultados. Os resultados evidenciam que: i) para - radiologista tomar uma decisão é necessário articular os conhecimentos declarativo, procedural e condicional; ii) os radiologistas classificados como especialistas possuíam pelo menos 8 anos de trabalho, desde sua especialização em diagnóstico por imagem, o que se aproxima à regra dos dez anos de preparação para se tornar especialista, proposta no estudo de Ericsson e Lehmann (1996); iii) nem todo radiologista consegue alcançar a perícia; iv) baseado na taxonomia de Bloom et al. (1956) foram identificadas e descritas seis categorias para radiologistas. As recomendações para práticas futuras decorrentes desse estudo incluem: aumentar as atividades de aprendizagem ativa, em que a realização da atividade é colaborativa entre novato(s) e especialista(s); expor os novatos a diagnósticos desafiadores e compartilhar com especialistas a elaboração de diagnóstico para incentivar a reflexão; estabelecer ambientes seguros de aprendizagem, em que os erros são compreendidos como oportunidade de aprendizagem.

Palavras-chave: Conhecimento. Aprendizagem. Perícia. Organizações intensivas em Conhecimento.

\section{Evolutionary categories for radiologists to gain expertise}

\begin{abstract}
The objective of the article is to identify and describe the evolutionary categories for a radiologist to gain the expertise. The study is supported by Bloom's et al. (1956) learning theory, which allows us to verify learning outcomes. From a qualitative study, the learning process of novice radiologists and specialists is presented and explained every step necessary to become a specialist in an area of diagnostic imaging. A total of 43 radiology and diagnostic imaging organizations participated in the study, of whom 21 were specialists and 28 were newcomers. The data were collected through observation, semi-structured interviews and result verification. The results show that: i) for the radiologist to make a decision, it is necessary to articulate declarative, procedural and conditional knowledge; ii) radiologists classified as specialists had at least 8 years of work, from their specialization in diagnostic imaging, which is close to the rule of ten years of preparation to become a specialist, proposed in the study by Ericsson and Lehmann (1996); iii) not every radiologist can achieve the skill; iv) based on Bloom's taxonomy et al. (1956), six categories were identified and described for radiologists. Recommendations for future practice arising from this study include: increasing active learning activities, where the activity is collaborative between beginner (s) and specialist (s); expose newcomers to challenging diagnoses, and share diagnostics with experts to encourage reflection; establish safe learning environments, where mistakes are understood as a learning opportunity.
\end{abstract}

Keywords: Knowledge. Learning. Expertise. Knowledge-intensive organizations. 


\section{INTRODUÇÃO}

O diagnóstico por imagens tornou-se tão essencial para a prática da medicina que é difícil conceber qualquer segmento da profissão sem a assistência fornecida pelo uso de imagens. Segundo Patti et al. (2008, p.1042) "estima-se que $40 \%$ dos encontros entre médico e paciente resultam na indicação do uso de imagens para diagnóstico ou revisão de estados clínicos". Sendo assim, o diagnóstico por imagem é uma ferramenta de apoio à prática clínica, que pode ser utilizado em contextos variados, o que inclui tanto o diagnóstico de doenças, como sua avaliação.

A atividade de diagnóstico é caracterizada como intensiva em conhecimento, pois é altamente especializada (MILES, 2005), possui forte dependência de conhecimento profissional, complexidade de operação e dificuldade de automatização (ISIK; MERTENS; VAN DEN BERGH, 2013). Sendo uma atividade altamente complexa com componentes cognitivos e perceptivos muito exigentes, requer que o radiologista desenvolva a capacidade de executar o processo com precisão e eficiência (JORRITSMA; CNOSSEN; VAN OOIJEN, 2015).

Além disso, pesquisas apontam que grande parte da tomada de decisão nas organizações de saúde é baseada em prática e depende fortemente da experiência e do conhecimento do profissional que irá realizar o diagnóstico (DORAN; SIDANI, 2007; PIZZI, 2009). Sendo assim, a imaginologia médica se tornou um componente crucial no processo de tomada de decisões e no cuidado de pacientes (KIM; MANSFIELD, 2014). A radiologia diagnóstica é então, uma subárea da radiologia que se concentra no diagnóstico, e pode definir o tipo de conduta futura a ser utilizada pelo médico solicitante do exame. Dessa forma, é clara então, a importância da competência do radiologista para um diagnóstico assertivo.

Dentre as competências que os especialistas em radiologia precisam desenvolver está a capacidade de reconhecer padrões visuais complexos (FITZGERALD, 2005). Esta competência se desenvolve com o passar dos anos, ao longo do exercício contínuo dessa atividade, desde a fase de aprendizado até que sejam atingidas as modalidades mais aperfeiçoadas de diagnóstico durante a prática profissional. Porém, existe escassez na literatura sobre como ocorre o processo de aprendizagem de radiologistas especialistas (GUNDERMAN; CHAN, 2003; WILLIAMSON et al. 2004).

Ao longo das últimas décadas, o campo da psicologia cognitiva fez um esforço para melhorar a compreensão de quais atividades de desenvolvimento realmente permitem melhorar o desempenho do indivíduo até atingir a perícia (ERICSSON; SMITH, 1991; STERNBERG; GRIGORENKO, 2003; CHI; GLASER; FARR, 2014.). Esses estudos indicam que o desempenho superior depende do desenvolvimento de ferramentas e estratégias cognitivas desenvolvidas durante um longo período de melhoria contínua, e habilitadas por meio de um compromisso proposto e sustentado (ERICSSON, 2006).

Segundo Ericsson e Lehmann (1996) a experiência é necessária para alcançar a perícia, porém, não é suficiente para conquistar um desempenho de especialista. Isso se deve ao fato que a maioria das atividades profissionais mais comuns proporcionam oportunidades limitadas para a melhoria das habilidades e aprendizagem efetiva. Essas condições não são propícias para estimular a prática deliberada que produz um desenvolvimento e crescimento sustentado (PETTY; SCHOLES; ELLIS, 2011).

Considerando a importância da assertividade do diagnóstico por imagem e as dificuldades existentes no aprendizado do mesmo, o esclarecimento de como melhorar a instrução de radiologistas especialistas é algo significativo.

Sendo assim, o objetivo deste estudo é identificar e descrever as categorias evolutivas para um radiologista conquistar a perícia, respaldado na teoria de aprendizagem de Bloom et al. (1956), que permite verificar os resultados de aprendizagem. Também permite conhecer o que pode ser mais eficaz quando se trata de compreender a imagem radiológica. Esse conhecimento pode então ser aplicado a futuros radiologistas, e servir como orientação para a melhorar a compreensão da aprendizagem diagnóstica.

Nesse sentido, este artigo contribui para melhorar o entendimento do processo de aprendizagem de radiologistas. Primeiro, a criação da escada evolutiva foi apoiada em uma taxonomia de complexidade dos objetivos educacionais (BLOOM et al., 1956), e essa taxonomia ocorre e é aplicada para descrição da evolução de conhecimento do radiologista perito. Em segundo lugar, o estudo revela os recursos de aprendizagem que 
os radiologistas identificaram como efetivos. Em terceiro lugar, o estudo fornece evidências empíricas a serem exploradas por estudos futuros. Em quarto lugar, os passos identificados, em cada categoria na escada evolutiva pelos participantes, podem servir de orientação para uma investigação mais aprofundada sobre a melhoria da educação em radiologia diagnóstica. Em quinto lugar, os resultados evidenciados em cada categoria subsidiam os líderes para ações de aprendizagem mais efetivas. Finalmente, proporcionam uma base que poderá incentivar estudos futuros sobre aprendizagem radiológica no diagnóstico baseado em evidências empíricas.

\section{DESENVOLVIMENTO}

Inicialmente serão apresentados os conceitos de compartilhamento do conhecimento e desenvolvimento organizacional, a posteriori o entrelaçamento entre taxonomia de Bloom e aprendizagem.

\subsection{Compartilhamento do conhecimento e desenvolvimento organizacional}

O conhecimento individual é transformado em conhecimento organizacional por meio do compartilhamento, interações entre um grupo de indivíduos, discussões, experiências e observações que permitam a criação de entendimentos. Ipê (2003) complementa ainda que o compartilhamento é o processo que permite que o conhecimento possuído por indivíduos seja convertido em um formato que possa ser entendido, absorvido e usado por outros indivíduos.

Dessa forma, a interação contínua e dinâmica suporta o fluxo do conhecimento explícito e tácito, e é a base para a criação do conhecimento organizacional. Assim, o conhecimento é essencialmente criado, compartilhado e implementado por meio das interações sociais. Nonaka e Takeuchi (1995) propuseram um modelo de criação do conhecimento, o modelo SECl. Esse modelo é um dos mais conhecidos a este respeito e consiste em quatro modos de conversão do conhecimento: socialização, externalização, combinação e internalização do conhecimento (NONAKA; TAKEUCHI, 1995).

O fluxo de conhecimento ocorre então entre esses modos de conversão. A socialização permite que o conhecimento possa ser criado e compartilhado. Porém, a externalização permite que o conhecimento seja disseminado de forma significativa na organização. Esse modo é essencial, já que o conhecimento explícito é de mais fácil difusão, ampliando-se a interação dos conhecimentos tácitos e explícitos.

Ainda em relação ao conhecimento, o cognitivismo apresenta três tipos de conhecimento: 1) declarativo (o que); 2) procedural (como); e 3) condicional (quando e por quê?) (STERNBERG, 2000). Marzano et al. (1988) e Giacomini, Silva e Greco (2011) postulam que, para que o indivíduo realize com competência uma atividade, é preciso a combinação desses três tipos de conhecimentos.

O conhecimento declarativo permite entender e compreender; corresponde ao conhecimento teórico que, em algum momento, foi um saber; é mais estático do que dinâmico e, para permitir a ação, necessariamente, precisa ser transformado em procedimentos (conhecimentos procedurais) ou em condições (conhecimentos condicionais), por si só não permite agir (MARZANO et al., 1988). Smith e Ragan (1999) afirmaram que, embora a aquisição do conhecimento declarativo seja, muitas vezes, mencionada como aprendizado de nível mais baixo, é fundamental para grande parte do pensamento humano e, geralmente, é adquirida dentro de estruturas significativas. O conhecimento declarativo é uma parte crítica do aprendizado de um indivíduo ao longo de sua vida. Para aprender diferentes tipos ou regras de conhecimento, o indivíduo deve, primeiramente, possuir conhecimento declarativo, um pré-requisito essencial para uma aprendizagem eficaz e superior. Para que ocorra a aprendizagem do conhecimento declarativo, o processo de aprendizagem deve incluir três atividades: ligar, organizar e elaborar. A primeira atividade para aprender novos conhecimentos declarativos, de forma eficaz, corresponde a vincular o novo conhecimento ao conhecimento existente ou a esquemas do indivíduo (JONASSEN; GRABINGER, 1990). A segunda, aglomerar e subordinar o novo conhecimento, pode simplificar a carga cognitiva (SMITH; RAGAN, 1999) e a terceira, individualizar novos conhecimentos de acordo com a experiência do indivíduo, tornando-os mais significativos. 
O conhecimento procedural consiste em conhecimentos dinâmicos que devem ser seguidos (sequência de ações), permitindo que o indivíduo realize determinada ação. São desenvolvidos, exclusivamente, na ação, na prática, pela experiência (TARDIF, 1997). Porém, para que um indivíduo seja competente, precisa ter os dois tipos de conhecimentos (declarativo e procedural). Esse tipo de conhecimento é mais sofisticado que o conhecimento declarativo, e condicional em termos cognitivos (envolvem tanto o conhecimento declarativo como o conhecimento condicional). De acordo com Schunk (1996), conhecimento procedural consiste em conceitos, regras e algoritmos. É o conhecimento de como realizar atividades cognitivas e, muitas vezes, é implícito. Ele se origina na atividade de resolução de problemas, em que um objetivo é decomposto em submetas para solucionar o problema (ANDERSON, 1995). Smith e Ragan (1999) afirmaram que as regras processuais são uma série "generalizável" de etapas iniciadas em resposta a uma classe particular de circunstâncias para alcançar um objetivo especificado e dizer ao indivíduo que certas ações devem ser tomadas.

O conhecimento condicional está ligado ao contexto da situação, quando e por quê e "descreve as circunstâncias de aplicação dos procedimentos" (KRATOCHVIL, 2009, p. 218). Também envolve uma rede de sequências de ação de condição. Corresponde, essencialmente, a classificações, a categorizações, são responsáveis pela transferência das aprendizagens, criam a perícia dos indivíduos especialistas e fornecem suporte para a flexibilidade pessoal e profissional. Consiste em "se então" ou "declarações de ação de condição"; estas declarações descrevem a relação entre dois ou mais conceitos. Smith e Ragan (1999) afirmaram que o conhecimento condicional permite ao indivíduo prever o que acontecerá se uma das variáveis, qualquer condição ou ação, for alterada. Para aprender o conhecimento condicional, os indivíduos devem, primeiramente, determinar as variáveis ou os conceitos envolvidos na situação e, em seguida, decidir sobre as regras aplicáveis a essa situação. Uma vez conhecendo as variáveis, as desconhecidas serão identificadas, e o efeito de variáveis conhecidas em variáveis desconhecidas deverá ser determinado. No final da sequência, da condição de ação, os indivíduos devem chegar a uma conclusão sobre a situação. Esse tipo de conhecimento ajuda os indivíduos a preverem, explicarem ou controlarem circunstâncias.

Para resolver os problemas, os indivíduos podem, simultaneamente, selecionar e aplicar o conhecimento condicional e processual, e aplicar as regras relacionadas. Nesse processo, os indivíduos também devem recordar o conhecimento declarativo, relacionado a essas regras. Quando os indivíduos empregam tanto o conhecimento condicional e procedural para resolver um problema ou para chegar a uma conclusão, o processo de aprendizagem em que estão envolvidos é chamado de ordem superior de aprendizagem, ou regra de resolução de problemas (SMITH; RAGAN, 1999). Schunk (1996) afirmou que a distinção entre os três tipos de conhecimento é importante em termos de suas implicações para a aprendizagem. As deficiências de diferentes tipos de conhecimento não só dificultam a aprendizagem, mas também produzem baixa autoeficácia dos indivíduos.

Para Bansal (2001), em organizações de radiologia o maior fluxo de conhecimento que contribui para o desenvolvimento da organização é gerado pelo conjunto dos conhecimentos dos radiologistas que realizam o diagnóstico das imagens.

Como já explicado, organizações de radiologia e diagnóstico por imagem são caracterizadas como organizações intensivas em conhecimento, devido ao seu tipo de atividade ser altamente cognitiva. Esse tipo de organização, sem dúvida, precisa de mais conhecimento para solucionar eficazmente problemas que podem ser muito complexos. Sendo assim, identificar os passos necessários para que os indivíduos da organização alcancem a perícia é algo relevante, pois as organizações só aprendem por meio de seus indivíduos que aprendem (HAYES; ALLINSON, 1998; VAN DE WIEL; SZEGEDI; WEGGEMAN 2004; SENGE, 1990). Essa visão é compartilhada por Simon (1991, p. 125) que afirma que "toda aprendizagem ocorre dentro de cabeças humanas individuais, uma organização aprende de duas maneiras: (a) pela aprendizagem de seus indivíduos, ou (b) adquirindo novos indivíduos que possuem o conhecimento necessário". Sendo assim, são os indivíduos que aprendem por meio de processos cognitivos e, quando esta aprendizagem é compartilhada, contribui para aprendizagem da organização, que é considerada uma das principais fontes de vantagem competitiva no contexto da gestão estratégica (CAMPBELL; ARMSTRONG, 2013). 
O processo de aprendizagem na organização é descrito como um sistema hierárquico composto por três níveis inter-relacionados: nível individual, nível de grupo e nível organizacional. A aprendizagem ocorre em indivíduos quando esses interagem e recebem novos conhecimentos de seu ambiente por meio de um fluxo de conhecimento, dentro ou fora da organização. Dessa forma, ao identificar o processo de aprendizagem dos indivíduos, a organização pode acelerar o novo processo de criação de conhecimento.

Neste contexto, utilizou-se nesta pesquisa a taxonomia proposta por Bloom et al. (1956), de forma a representar o processo de aprendizagem individual, suportando o planejamento, organização e domínio dos objetivos de aprendizagem.

\subsection{Taxonomia de Bloom e aprendizagem}

A perícia é definida como a capacidade de reproduzir consistentemente um desempenho superior em termos absolutos ou normativos. $O$ foco das pesquisas sobre especialistas é duplo: 1) ampliar a visão sobre quais elementos cognitivos são necessários para permitir um desempenho consistentemente superior; e 2) melhorar a compreensão de como os elementos cognitivos se desenvolvem. A pesquisa até o momento estabelece que a experiência se desenvolve ao longo de um longo período de engajamento proposital sustentado (ERICSSON; KRAMPE; TESCH-ROMER, 1993; ERICSSON; CHARNESS, 1994). Durante esse período, os indivíduos que alcançam sucesso na construção de sua experiência apresentam um padrão de progresso incremental, identificando e melhorando suas fraquezas. Esse processo de transformação de experiências em conhecimento é chamado de aprendizagem. Vários pesquisadores defendem que o saber é consequência da organização das capacidades cognitivas, como Bloom et al. (1956).

Dessa forma, a aprendizagem da atividade de diagnóstico por imagem deve atender requisitos que ajudem ao novato a alcançar melhores resultados. Entretanto, se estes não foram bem definidos, os resultados da aprendizagem poderão ser limitados.

A taxonomia de Bloom et al. (1956) foi fruto de um requerimento da associação Norte-Americana de Psicologia, que solicitou a seus membros que por meio de uma força tarefa discutissem, definissem e criassem uma taxonomia para atender aos objetivos de processos educacionais. Segundo Guskey (2001), Bloom et al. (1956), Bloom (1972), Anderson et al (2001) e Braun e Clark (2006) de forma geral, as características principais de cada um desses domínios (ou categorias) educacionais ou de aprendizagem, são:

a) Cognitivo (habilidades mentais): envolve aprender e ter domínio de um conhecimento. Adquirir e desenvolver novos conhecimentos, habilidades e atitudes. Esse domínio possui seis categorias (Conhecimento; Compreensão; Aplicação; Análise; Síntese; e Avaliação). As categorias podem ser consideradas como graus de dificuldades. Ou seja, os primeiros devem ser dominados antes que o próximo possa ocorrer.

b) Afetivo (emoções): envolve sentimentos e atitude. São as categorias emocionais e afetivas. As categorias são receptividade; Resposta; Valorização; Organização; e Caracterização.

c) Psicomotor (habilidades manuais ou físicas): consiste nas habilidades físicas do indivíduo. Porém, Bloom não definiu uma taxonomia para esse domínio.

Dos três domínios apresentados (cognitivo, afetivo e psicomotor), o domínio cognitivo é o mais conhecido e utilizado. Muitos instrutores utilizam esses pressupostos teóricos para definir seu planejamento, objetivos, estratégias e formas de avaliação. Segundo Anderson et al. (2001) o processo cognitivo é o meio que permite que o conhecimento seja alcançado ou construído e utilizado para resolução dos problemas diários e eventuais (ANDERSON et al., 2001).

A taxonomia de Bloom et al. (1956) pode ser pensada como os objetivos do processo de aprendizagem. Ou seja, após um episódio de aprendizado, o indivíduo deve ter adquirido uma nova habilidade, conhecimento e/ou atitude. 


\section{METODOLOGIA}

Essa é uma pesquisa aplicada, pois colabora para fins práticos (DE ANDRADE; MARCONI; LAKATOS, 2007). Quanto à abordagem do problema, trata-se de uma pesquisa qualitativa, com o intuito de identificar e descrever as categorias evolutivas para um radiologista conquistar a perícia, respaldado na teoria de aprendizagem de Bloom et al. (1956), mostrando os resultados de aprendizagem dos radiologistas. É descritiva, pois se propõe a conhecer e interpretar a realidade sem nela interferir (CHURCHILL; IACOBUCCI, 2006). Esse tipo de pesquisa utiliza como forma de estudo, técnicas que contemplam interpretação para poder descrever e traduzir todos os elementos que compõem os dados pesquisados. Com esse tipo de pesquisa, buscou-se obter dados descritivos sobre a temática em questão, a partir dos dados coletados com os entrevistados. Como procedimento técnico, utilizou-se um estudo multicaso, que segundo Triviños (1987), refere-se a um estudo de caso aplicado em mais de uma população.

O universo de pesquisa foi o de três organizações de radiologia e diagnóstico por imagem de Santa Catarina. Os exames realizados nas três organizações variam entre exames de rotina aos altamente especializados. Tais organizações são identificadas nesse artigo como 01 (Organização 1), O2 (Organização 2) e O3 (Organização 3). Especificamente, participaram 43 radiologistas pertencentes a essas organizações.

Os sujeitos da pesquisa foram classificados como novatos e especialistas. Para realizar essa classificação, foram adotados os critérios sugeridos por Dreyfus e Dreyfus (1980): a) novato: rígida aderência a regras e sem julgamento discricionário; b) especialista: não se baseia em regras ou orientações, compreensão intuitiva de situações com base em profundo conhecimento tácito.

Para complementar essa classificação, foram observados outros aspectos, como: ano de treinamento em que se encontra, existência de subespecialização na área de diagnóstico, especialidade adicional, tempo de formado, tempo de trabalho na área de diagnóstico e tempo de atuação na organização atual. Durante as observações, identificou-se que os radiologistas especialistas eram reconhecidos como tal por parte dos outros radiologistas do grupo.

Para selecionar as organizações participantes da pesquisa, considerou-se como primeiro critério, possuir pelo menos dez anos de atividade e fazer uso do sistema de comunicação Picture Archiving and Communication System (PACS), que consiste em um sistema de comunicação e arquivamento de imagens.

Os sujeitos da pesquisa foram médicos radiologistas novatos e especialistas com especialização em radiologia e diagnóstico por imagem. Justifica-se essa escolha pelo fato desses atores permitirem a identificação das diferentes categorias de aprendizagem na taxonomia de Bloom.

A pesquisa foi aplicada entre os meses de junho de 2016 a janeiro de 2017, conforme a disponibilidade dos entrevistados. Inicialmente, foram realizadas observações por um período de 15 (quinze) dias em cada um dos três grupos participantes, com o objetivo de familiarizar-se com o ambiente e observar o processo de interação dos atores em seu ambiente natural de trabalho.

Na sequência, foram realizadas entrevistas com o auxílio de um roteiro semiestruturado, organizado a partir do referencial teórico. Os assuntos do roteiro abordaram questões relativas às percepções sobre: a) diferenças entre novatos e especialistas; $b$ ) formas efetivas de aprendizagem; c) principais formas de avaliação da aprendizagem (DOROW, 2017). Realizaram-se 43 (quarenta e três) entrevistas, sendo 21 (vinte e uma) com o primeiro grupo, 12 (doze) com o segundo grupo e mais 10 (dez) com o terceiro grupo nas três organizações estudadas. Essas entrevistas foram gravadas e transcritas de forma integral. Utilizou-se a análise temática como técnica de análise dos dados originados das entrevistas (BRAUN; CLARKE, 2006).

Com base nos resultados da análise elaborou-se um questionário, de forma a reduzir a intersubjetividade de entendimento dos autores. O questionário era composto por afirmativas fechadas que refletiam os resultados relevantes identificados na análise temática. Em cada afirmativa, o radiologista tinha uma escala Likert para assinalar o quanto concordava com a afirmativa em questão (de 'discordo totalmente' até 'concordo totalmente'). Optou-se por utilizar uma escala ímpar - Likert-5, para evitar o viés da não existência de ponto médio, conforme indicam algumas pesquisas relevantes (DARROCH, 2005; INKINEN, KIANTO; VANHALA, 2015). 


\section{RESULTADOS E DISCUSSÃO}

Nesta seção serão apresentados e discutidos os resultados encontrados na pesquisa de campo, a partir de análises com as categorias delineadas.

\subsection{Diagnóstico por imagem: articulação dos conhecimentos}

Durante as observações, identificou-se a manifestação dos três tipos de conhecimento (declarativo, procedural e condicional) na prática do diagnóstico por imagem. As características do conhecimento do radiologista, bem como suas definições e forma de aquisição são apresentadas no Quadro 1.

Quadro 1- Características e definições dos conhecimentos do radiologista

\begin{tabular}{|c|c|c|}
\hline $\begin{array}{c}\text { Tipos de } \\
\text { conhecimento }\end{array}$ & Definições & Aquisição \\
\hline $\begin{array}{l}\text { Conhecimento } \\
\text { declarativo }\end{array}$ & $\begin{array}{l}\square \text { Conhecimento médico que envolve as } \\
\text { considerações, indicações, contraindicações, } \\
\text { finalidade, riscos e limitações sobre os } \\
\text { exames. } \\
\square \text { Definições anatômicas, sinais e sintomas } \\
\text { fisiológicos, características das doenças e } \\
\text { sua manifestação visual, bem como a } \\
\text { terminologia própria. }\end{array}$ & $\begin{array}{l}\text { Pela leitura de livros, artigos e } \\
\text { outros materiais na web, onde se } \\
\text { encontram explicitadas } \\
\text { informações sobre anatomia e } \\
\text { fisiologia ou pela participação } \\
\text { em aulas e qualquer tipo de } \\
\text { evento onde os indivíduos } \\
\text { externariam seu conhecimento. }\end{array}$ \\
\hline $\begin{array}{l}\text { Conhecimento } \\
\text { procedural }\end{array}$ & $\begin{array}{l}\square \text { Conhecimento que permite sistematizar } \\
\text { os procedimentos, passo a passo. } \\
\square \text { Sequência de argumentação necessária ao } \\
\text { diagnóstico de uma imagem. } \\
\square \text { Correlacionar os sinais e sintomas com os } \\
\text { achados da imagem. }\end{array}$ & $\begin{array}{l}\text { Pela prática (experiência), } \\
\text { fazendo ou observando um } \\
\text { radiologista desempenhar suas } \\
\text { ações já sistematizadas e } \\
\text { externalizadas sobre um } \\
\text { procedimento. }\end{array}$ \\
\hline $\begin{array}{l}\text { Conhecimento } \\
\text { condicional }\end{array}$ & $\begin{array}{l}\square \text { Conhecimento que envolve a capacidade } \\
\text { de associação para reconhecer padrões } \\
\text { visuais. } \\
\square \text { Distinguir imagens sobrepostas de } \\
\text { anormalidades. } \\
\square \text { Diferenciar o que é principal do que é } \\
\text { complementar. } \\
\square \text { Saber como e porque algo está se } \\
\text { manifestando ou não na imagem. } \\
\square \text { Entendimento que permite julgar a } \\
\text { qualidade de uma imagem e a necessidade } \\
\text { de solicitar outro exame. }\end{array}$ & $\begin{array}{l}\text { Pela experiência prática de } \\
\text { visualização e categorização de } \\
\text { centenas ou milhares de } \\
\text { imagens médicas (normais e } \\
\text { anormais) e em diferentes } \\
\text { formatos que, em alguns casos, } \\
\text { podem vir acompanhadas do } \\
\text { feedback de outro radiologista } \\
\text { mais experiente. }\end{array}$ \\
\hline
\end{tabular}

Fonte: os autores (2017)

Percebe-se que o primeiro conhecimento aprendido é o conhecimento declarativo, que consiste na base adquirida durante a faculdade de medicina, habilitando o radiologista a correlacionar seu conhecimento de visualização de imagens (condicional), por meio da correlação entre estes conhecimentos (declarativo e condicional), utilizando seu conhecimento procedural.

A articulação desses três tipos de conhecimentos representa a base da formação de competência do radiologista, necessária para a tomada de decisão. Um diagnóstico preciso é resultado do reconhecimento proficiente de padrões na imagem. Com base no seu conhecimento (declarativo) de categorias da patologia que melhor se adapta às características relevantes identificadas na imagem (condicional) define-se a provável doença. Exemplificando-se na fala de um dos entrevistados: 
Tem uma bagagem que é fundamental; reconhecer os padrões da imagem e relacionar com as características dos sintomas. Por exemplo, um radiologista realizar um exame de tomografia abdominal para verificar cálculo vesicular é inadmissível, pois se ele conhece as particularidades de cada modalidade vai saber que a sensibilidade e acurácia da tomografia é menor do que do ultrassom, sem falar no preço que é menor, a aquisição é mais rápida e não vais expor o paciente à radiação (O3E10).

A análise das entrevistas, permitiram a construção da Figura 1, que ilustra a fala anterior do radiologista em relação à articulação desses conhecimentos para a resolução do diagnóstico.

Figura 1- Exemplo da articulação do conhecimento declarativo, procedural e condicional para resolução do diagnóstico

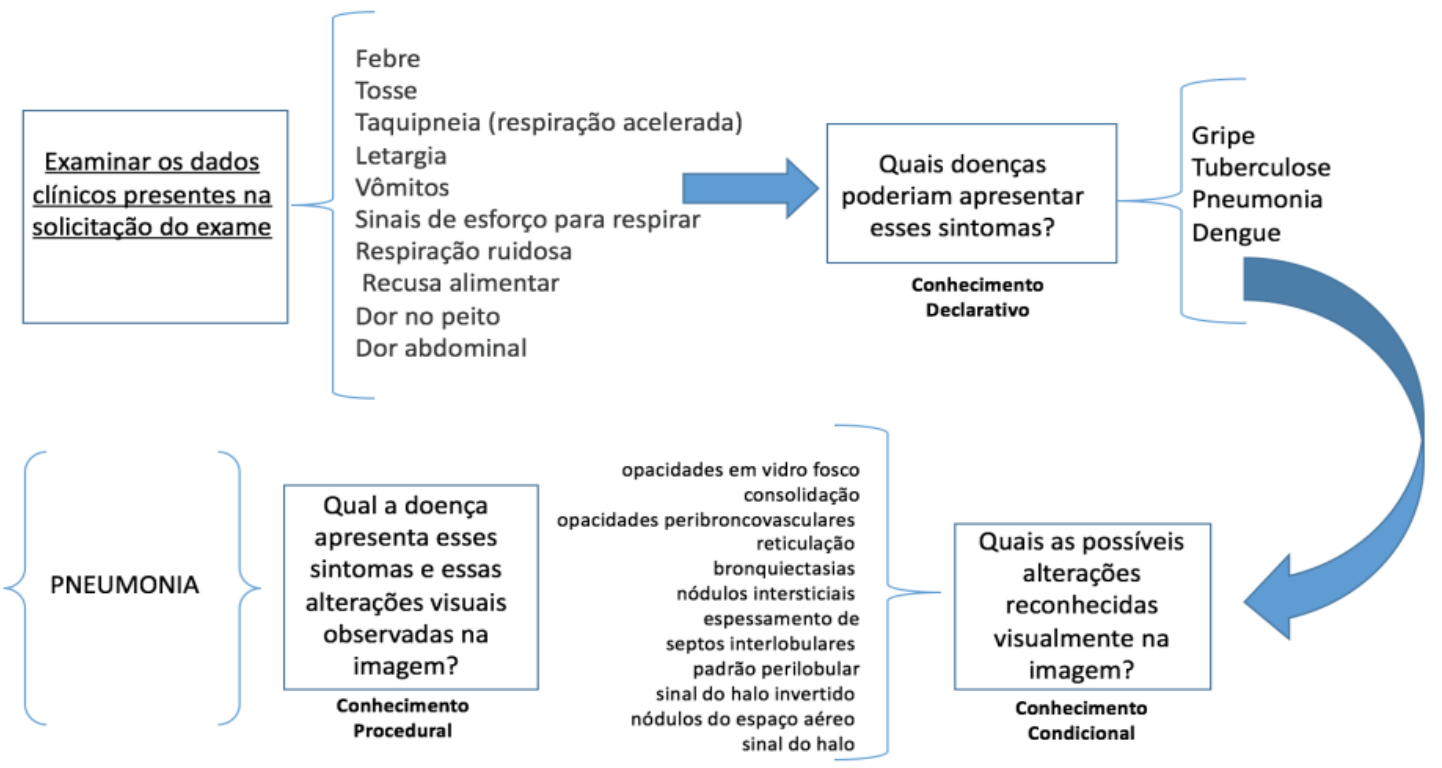

Fonte: DOROW (2017)

Conforme demostrado, o conhecimento declarativo é a base para que o radiologista conheça as doenças e os sinais e sintomas que o paciente pode apresentar. A partir dessas informações é iniciada a formulação das hipóteses diagnósticas. O conhecimento declarativo do indivíduo permite que ele tenha uma base para relacionar os sinais e sintomas evidenciados na solicitação do exame (conhecimento procedural) com as alterações reconhecidas visualmente na imagem e encontrar a conclusão diagnóstica (conhecimento condicional).

Caso haja uma lacuna em algum desses conhecimentos, inevitavelmente, em algum momento, ele sentirá dificuldades, ou não será capaz de realizar o diagnóstico com segurança. A maioria dos entrevistados explicou que, sem uma base de conhecimento declarativo completa e estruturada, não é possível se tornar um bom radiologista:

Sem um bom conhecimento teórico, a sua formação da clínica mesmo, você não vai sair do lugar, pode olhar a imagem o dia todo que o diagnóstico não vai sair como mágica, por isso a gente tem que estar estudando o tempo todo para atualizar e refinar a nossa base, isso é metade do caminho. (O1E4).

Portanto, enfatiza-se que o conhecimento declarativo do radiologista, resultado de uma boa formação acadêmica de radiologia e diagnóstico por imagem, é um conhecimento fundamental para ser um bom profissional. Outro tipo de conhecimento que separa bons de ótimos radiologistas é o conhecimento condicional, que compreende o reconhecimento de padrões visuais. De acordo com cada modalidade de diagnóstico, esse conhecimento é alterado, pois a imagem se apresenta em padrões visuais diferentes. Esse conhecimento condicional, representado pelo reconhecimento de padrões visuais, foi definido pelos radiologistas como diferenciador. 
O conhecimento condicional permite o reconhecimento de informações anatômicas e fisiológicas, nas diversas modalidades de imagens: raios $\mathrm{X}$, tomografia computadorizada, mamografia, ultrassom, densitometria, entre outras. Porém, realizar diagnósticos em cada uma dessas modalidades exige muita prática deliberada, e leva tempo para que cada novo padrão possa ser reconhecido.

Como a variação de padrões de doenças é grande, o conhecimento para reconhecer e categorizar um amplo leque de possíveis aparências visuais é, então, uma parte essencial e singular que diferencia os radiologistas. Dominar as habilidades periciais do diagnóstico perceptivo é o objetivo da aprendizagem crítica durante o progresso que envolve estágios de percepção e construção das habilidades relacionadas ao diagnóstico.

Por meio das entrevistas, foi possível identificar que a prova escrita é uma forma para testar o conhecimento declarativo do radiologista, ainda na residência. A prova oral avaliava a capacidade perceptiva em relação a cada estrutura e seu padrão visual correspondente. A competência do conhecimento procedural do indivíduo era testada por meio da explicitação entre as correlações que o radiologista fazia, avaliando o que via e reconhecia na imagem, e definindo significados (alterações) que essas características poderiam representar.

Sendo assim, observa-se que o desenvolvimento do conhecimento pode ser mensurado por testes escritos. Porém, somente possuir uma base maior de conhecimento declarativo não significa ser um radiologista melhor. Por isso é que as outras habilidades de detecção e interpretação da imagem diagnóstica também são avaliadas, como a prova oral, que foi comentada pelo entrevistado.

Para alcançar a competência em diagnosticar imagens o radiologista conta com uma massa crítica de conhecimento, adquirida, principalmente, por meio da formação (conhecimento declarativo) e, posteriormente, reforçado pela experiência de trabalho (conhecimento procedural e condicional), melhorando, assim, sua base de conhecimento ao longo dos anos.

Dessa forma, a atualização e aquisição dos novos saberes e competências ocorrem basicamente de duas formas: a) por meio da consolidação de padrões visuais e do constante reconhecimento de novos padrões, aliados à reflexão das possíveis atitudes que possam apoiar a tomada de decisão e b) por meio da ruptura de estruturas previamente estabelecidas. Isso resulta da integração do conhecimento prévio aliado a observações repetidas de novas e diferentes imagens. Ainda conforme evidenciaram os entrevistados, a velocidade de tradução do conhecimento declarativo, procedural e condicional em competências próprias para agir, aumenta quando o radiologista recebe feedback e pratica intensamente (resolvendo individual e repetitivamente os diagnósticos). $O$ parecer de um especialista sobre o trabalho ajuda os novatos a desenvolverem estratégias cognitivas mais ricas de automonitoramento do conhecimento.

Nos três grupos estudados, os radiologistas que contemplaram os critérios - para serem classificados como especialistas, conforme indicado na metodologia, possuíam, pelo menos, 8 anos de trabalho, após a especialização em diagnóstico por imagens.

Apresentados os tipos de conhecimento dos radiologistas e a forma como esses são articulados para realizar o diagnóstico por imagem, são então identificadas e descritas as categorias evolutivas, que segundo Bloom et al. (1956), definem o desenvolvimento do radiologista especialista.

\subsection{Categorias de evolução do conhecimento de radiologistas}

A evolução do conhecimento do radiologista é representada com o suporte da taxonomia de Bloom que define seis categorias: a) adquirir conhecimento; b) compreender; c) aplicar; d) analisar, e) sintetizar; e f) avaliar (BLOOM et al., 1956).

O primeiro degrau (categoria) "adquirir conhecimento", representa a utilização das regras que são conhecidas, e demonstra a falta de reflexão do novato por falta de experiências. $O$ conhecimento clínico dos sinais e sintomas do paciente afeta diretamente a forma como o radiologista percebe e visualiza alterações na imagem.

A evolução do reconhecimento de padrões visuais é algo que ocorre de maneira particular com cada radiologista. A velocidade dessa evolução pode variar de acordo com: o tempo de trabalho, a repetibilidade, a 
quantidade, a complexidade dos diagnósticos realizados e o feedback recebido. Porém, o conhecimento dos sinais e sintomas do paciente afeta diretamente a forma como o radiologista percebe (visualiza) a imagem. Durante o desenvolvimento perceptivo (reconhecimento dos padrões na imagem), o radiologista aprende a reconhecer os recursos ou conjuntos de recursos pelos quais ele atribui categorias, como normal ou anormal. Complexidades de objetos ou sobreposições exigem a identificação das características salientes. Um conjunto específico de características implica em avaliar as variações e atribuir alta importância às associações ou ausências significativas para manter a precisão de definição.

Já no segundo degrau (categoria) "compreender", o novato passa a aproveitar as experiências que já conquistou até então, porém, nos casos difíceis, ainda demonstra dificuldade e insegurança.

Pode se inferir que os radiologistas com maior experiência possuem um repertório maior de características conhecidas, associações e níveis de semelhança ou diferença. Consequentemente, desenvolvem uma maior noção conceitual. A base de conhecimentos é atualizada gradualmente, na medida em que novas imagens vão sendo observadas. Dessa forma, quanto maior a frequência de observações, maior a percepção visual do radiologista para detectar diferentes padrões. A interpretação das características da imagem é considerada um elemento chave para um diagnóstico preciso, conforme justifica a fala de um entrevistado:

Depois de reconhecer algo na imagem é preciso correlacionar com o conhecimento que você possui sobre aquele tipo de característica visualizada e interpretar o que está vendo. Um radiologista com dificuldades para interpretar as características vai com certeza, apresentar um diagnóstico problemático. (O3E4).

Percebe-se que, para os especialistas visuais, associações complexas são facilmente reconhecidas, e a categorização se torna mais refinada. Eles reconhecem características que se identificam em níveis mais dependentes de categorização. Radiologistas mais experientes direcionam mais atenção para as características críticas, criam seus próprios critérios para ponderação e, consequentemente, as interrelações tornam-se mais fortes.

Outro ponto diferenciador observado para a interpretação de imagens está na maior base de conhecimento do radiologista experiente. O reconhecimento do problema, baseado em pistas familiares, leva diretamente ao insight de uma solução já praticada. Sendo assim, sua categorização se torna mais eficiente, e requer menos esforço. Questões como "o que estou vendo?", "o que isso significa?", "por que isso está acontecendo?", implicam em um talento para fornecer um significado que é retrospectivo, ou seja, o radiologista, dificilmente, consegue dar sentido às ações ou aos fatos que nunca ocorreram. Afinal, inevitavelmente, o diagnóstico presente é comparado com suas experiências pessoais passadas, com a finalidade de estabelecer um significado.

O terceiro degrau (categoria) "aplicar", representa a capacidade de criar novos procedimentos para realizar a ação, onde a prática deliberada Ihe permite a reflexão sobre as decisões a serem tomadas. Essa categoria é definida pelo desenvolvimento perceptivo mais refinado, que consiste no reconhecimento de padrões visuais, o radiologista aprende a reconhecer os recursos ou conjuntos de recursos pelos quais ele atribui categorias, como normal ou anormal. Complexidades de objetos ou sobreposições exigem a identificação das características salientes. Um conjunto específico de características implica em avaliar as variações e atribuir alta importância às associações ou ausências significativas para manter a precisão de definição.

No quarto degrau (categoria) "analisar", devido a reflexão realizada nas categorias anteriores, o radiologista é então, capaz de perceber formas inovadoras e criar novos caminhos para chegar à conclusão diagnóstica. É capaz então de distinguir sobreposições, classificar a qualidade da imagem e relacionar as evidências fornecidas pela imagem com sua hipótese diagnóstica.

O quinto degrau (categoria) "sintetizar", demanda alto nível de conhecimento, articulado em experiências reais do que funciona e do que efetivamente não funciona. Nessa categoria, o radiologista cria seu próprio estilo diagnóstico, integra e combina ideias em uma proposta de resolução de diagnóstico personalizada, que caracteriza suas próprias particularidades para tomada de decisão. 
Com experiência e aprendizagem, um radiologista especialista poderá usar alguns atalhos (diferentes formas de resolver o problema) para chegar ao diagnóstico final. Dessa forma, acaba tendo um esforço mental mais baixo para resolução, quando comparado a um novato. Os atalhos funcionam porque direcionam o radiologista a soluções bem-sucedidas, que já foram apreendidas anteriormente em circunstâncias semelhantes e funcionaram (na categoria de compreender).

Finalmente, o sexto degrau "avaliar", representa uma estruturada articulação dos conhecimentos, que permite facilidade e segurança para que o radiologista julgue as imagens em termos de evidências clínicas, aliadas ao reconhecimento de padrões visuais com base em padrões e critérios específicos. O resultado dessa avaliação é uma opinião crítica.

A velocidade na interpretação adequada de uma imagem é uma característica crucial que define um especialista. Essa alta velocidade de "avaliar" com critério as imagens demonstra a consolidação do processo de aprendizagem proposto por Bloom et al. (1956).

Cabe salientar que uma das formas de identificar a categoria de aprendizagem de um radiologista é por meio da prática de revisão por pares, que consiste na dupla assinatura dos diagnósticos. Dessa forma, os radiologistas mais experientes identificam possíveis falhas nos diagnósticos dos menos experientes e podem fornecer feedback e orientar ações que auxiliem a melhorar a aprendizagem dos colegas.

De forma geral, considera-se que reconhecer padrões visuais complexos e realizar o diagnóstico com base nos sinais e sintomas e nas informações visuais fornecidas pela imagem pode ser problemático, pois, embora as características sejam estruturas elementares que compõem e identificam o corpo humano, sua composição pode variar muito entre ossos, órgãos, tecidos e sua exata localização e tamanho, de acordo com os diferentes pontos de vista.

Aliado a essas variações, existe também uma grande diversidade de exames de imagem disponíveis para esclarecer cada caso em particular. Cada forma de representação da imagem apresenta um padrão visual diferente, desde imagens 2D a 3D. Por exemplo, a mesma estrutura (pulmão) pode ter diferentes representações visuais, de acordo com a tecnologia utilizada para aquisição da imagem. Dificilmente o radiologista será perito em diferentes subespecialidades específicas ao diagnóstico por imagem. A própria perícia em relação a diferentes modalidades de imagens dentro de um mesmo domínio é algo que requer uma grande base de conhecimento e prática deliberada. Conforme revela um entrevistado, algumas modalidades de imagem exigem alto nível de conhecimento: "O ultrassom é uma imagem de difícil compreensão, o exame é realizado em tempo real e exige do radiologista um alto nível de conhecimento e muita prática para fazer o laudo." (O1E3).

Conforme explicitado nas falas dos entrevistados, existem diferenças na base de conhecimento dos radiologistas, de acordo com sua subespecialização e com o tipo de padrão de imagem que estão habituados a ler como: raios $X$, tomografia computadorizada, ressonância magnética, mamografia e densitometria óssea. Cada uma dessas modalidades de imagem demanda uma base de conhecimento diferente para que seja interpretada.

A comparação mental de um novo diagnóstico com experiências anteriores foi uma estratégia bem definida pelos novatos até a categoria da compreensão. A reflexão e a capacidade de estruturar novas percepções sobre a imagem caracteriza a categoria de aplicar e segue com o desenvolvimento da percepção de novas formas de agir quando a utilização das experiências conhecidas não é suficiente para tomada de decisão caracterizando a categoria da análise. A identificação de uma conduta clara, que identifica o estilo diagnóstico do radiologista, representa a categoria da síntese que antecede a categoria final da avaliação, compreendida pela facilidade e agilidade no reconhecimento de padrões complexos e associação com sinais e sintomas do paciente, permitindo a consolidação da competência para fazer julgamentos críticos sobre a imagem.

Para que o radiologista possa atingir uma nova categoria na escada evolutiva da perícia, sua reflexão crítica deve estar voltada para suas atividades práticas de trabalho. Porém, a habilidade dessas reflexões pode ser alcançada mais rapidamente quando existem diálogos frequentes entre o radiologista orientador e 0 novato, seja no fornecimento de feedback construtivo, direcionamento, ou no fornecimento de estratégias mais eficazes de trabalho, que já foram experimentadas e validadas pelo radiologista orientador. 
Na Figura 2 são apresentadas as diferentes ações que o radiologista desenvolve na construção de seu conhecimento. A construção da Figura se baseou em Bloom et al. (1956).

Figura 2 - Escada evolutiva da perícia do radiologista

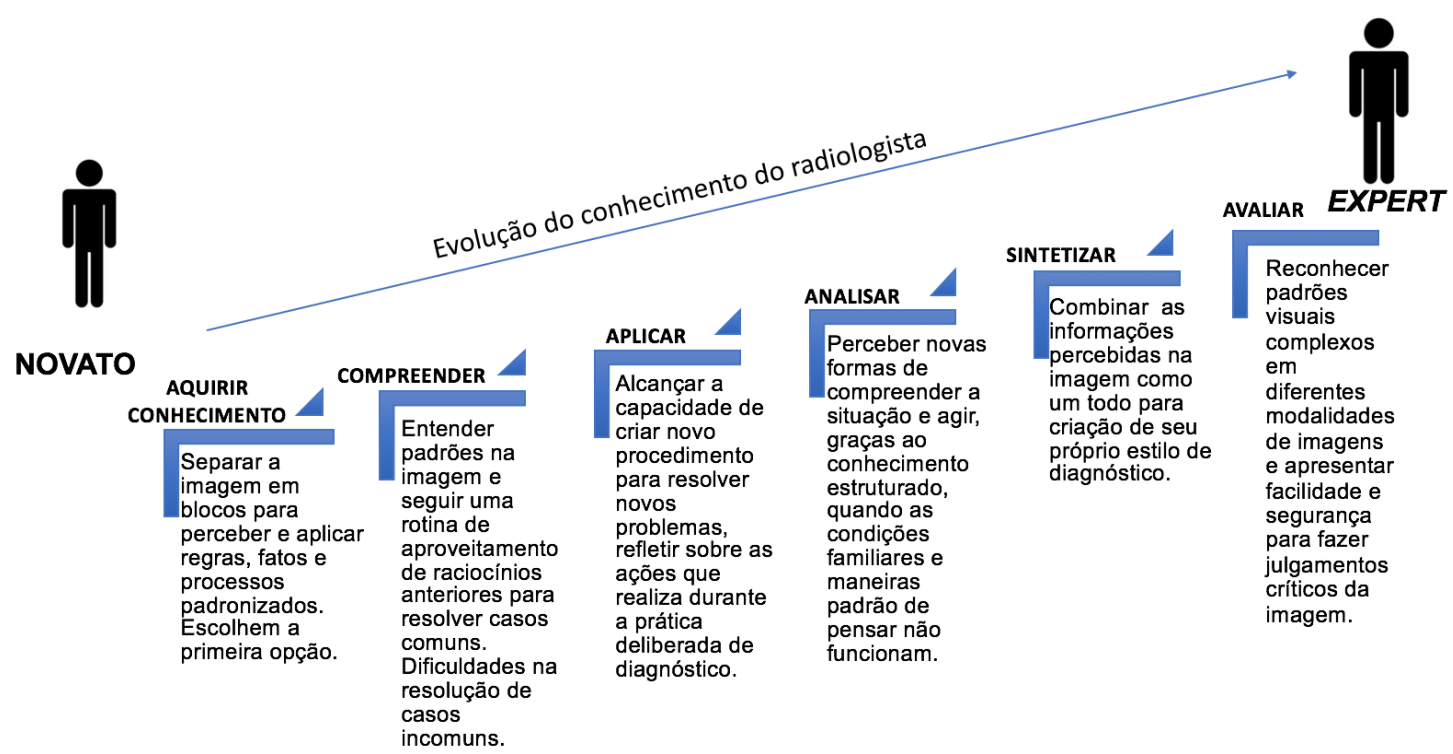

Fonte: DOROW (2017)

Resumindo, a forma como o conhecimento declarativo, procedural e condicional são registrados na memória por meio de experiências práticas, bem como as diferentes formas de agir desempenhadas pelos radiologistas especialistas, são decorrentes de sua base de conhecimento e experiência. Dessa forma, fica subentendido como é relevante a estruturação do conhecimento apropriado ao domínio específico, na formação cognitiva do radiologista, e a aplicação repetitiva desses conhecimentos por meio da experiência prática, para a internalização de novos conhecimentos de forma mais efetiva. O recebimento de um feedback pelos pares também foi um elemento diferenciador na criação de estruturas sólidas de conhecimento e formação de um modelo mental apropriado à resolução de problemas complexos. Além disso, a capacidade do especialista de tomar boas decisões está associada ao seu domínio de atuação, ser especialista em uma subespecialidade não garante que o radiologista também seja especialista em outras.

\section{CONSIDERAÇÕES FINAIS}

A falta de um planejamento educacional adequado, que defina conteúdos e estratégias eficazes, pode prolongar o tempo necessário para a evolução do conhecimento ou mesmo a perda de motivação na realização da atividade.

Esta pesquisa demonstra que a evolução do conhecimento do radiologista está relacionada a dois fatores: a) possuir conhecimentos: declarativo, procedural e condicional e b) articular (gerenciar) esses conhecimentos para tomada de decisão.

Observou-se que a utilidade de identificar as categorias necessárias a um domínio do conhecimento reside na capacidade de traduzir quais são os entendimentos individuais necessários para melhorar a competência do radiologista. Dessa forma, as organizações poderão desenvolver ações específicas condizentes ao desenvolvimento do conhecimento, tanto individual quanto organizacional. Isso permite que as organizações ganhem uma visão em várias perspectivas de seu ambiente, e respondam de forma coordenada às suas necessidades.

O compartilhamento de conhecimentos e experiências entre indivíduos com diferentes bases de conhecimento (novatos e especialistas) é benéfico para ambos, pois fornece exemplos aos novatos em relação 
forma de estruturar a tomada de decisão; e amplia a necessidade de aprendizagem dos especialistas, que ao ensinar os menos experientes, desenvolvem sua base de conhecimento.

Além disso, lidar com situações complexas também exige um constante processo de análise e reflexão acerca das experiências vividas. Um dos resultados da pesquisa é que a reflexão dos novatos se inicia quando esses encontram situações que não são familiares, pois incertas e inéditas, de modo que o conhecimento que possuem não lhes permite encontrar uma solução. Desse modo, a falta de conhecimento desperta o processo de reflexão necessário para ascender às categorias finais da taxonomia de Bloom. Quanto ao especialista, foi constatado que a prática por si só não permite o desenvolvimento do conhecimento. Para que o indivíduo possa evoluir nas categorias da taxonomia de Bloom também é necessário refletir sobre as decisões, de maneira a gerenciar e utilizar efetivamente os conhecimentos (declarativo, procedural e condicional).

Constatou-se que os radiologistas refletem pouco ao resolver casos conhecidos. Porém, diagnósticos desconhecidos e não familiares demandam uma maior procura pelo conhecimento necessário, o que leva ao maior compartilhamento de conhecimento e ao aumento do processo de reflexão. Sendo assim, situações desafiadoras e casos difíceis são indicadas para estimular o desenvolvimento das competências individuais e organizacionais.

A capacidade da organização de formular metas e modificá-las ao longo do tempo, monitorando a evolução do conhecimento dos indivíduos, são aspectos chaves para a aprendizagem organizacional. Em suma, conclui-se que a identificação das categorias (adquirir conhecimento, compreender, aplicar, analisar, sintetizar e avaliar) presentes no desenvolvimento do radiologista possui implicações importantes para a área da saúde. Quando uma organização não conhece e avalia a competência de seus profissionais é incapaz de gerenciar o conhecimento. Como consequência, a avaliação de intervenções alternativas, destinadas a promover a aprendizagem e o desempenho da organização, possivelmente estarão desalinhadas da real lacuna de conhecimento. Embora a identificação das lacunas de conhecimento seja um processo complexo, é fundamental para a organização sua identificação para o sucesso organizacional, e vitais para o progresso sustentado.

\section{REFERÊNCIAS}

ANDERSON, J. R. Cognitive psychology and their acquisition. Psychological Review, Washington, v. 94, n. 1, p. 192-210, 1995.

ANDERSON, Lorin W. et al. A taxonomy for learning, teaching and assessing: A revision of Bloom's taxonomy. New York. Longman Publishing. Artz, AF, \& Armour-Thomas, E.(1992). Development of a cognitive-metacognitive framework for protocol analysis of mathematical problem solving in small groups. Cognition and Instruction, v. 9, n. 2, p. 137-175, 2001.

ANDERSON, L. W. et. al. A taxonomy for learning, teaching and assessing: a revison of Bloom's Taxonomy of Educational Objectives. Nova York: Addison Wesley Longman, 2001. 336 p.

BANSAL, M. Knowledge management: capitalizing a hospital's assets. Express Healthcare Management. India's first newspaper for the health care business, 2001. Disponível em: <http://www.expresshealthcaremgmt.com/20010915/editorial2.htm> Acesso em: 18 jul. 2017.

BLOOM, B. S. et al. Taxonomy of educational objectives. New York: David Mckay, 1956. 262 p. (v. 1)

BLOOM, B. S. Innocence in education. The School Review, v. 80, n. 3, p. 333-352, 1972.

BRAUN, Virginia; CLARKE, Victoria. Using thematic analysis in psychology. Qualitative research in psychology, v. 3, n. 2, p. 77-101, 2006.

CAMPBELL, Timothy T.; ARMSTRONG, Steven J. A longitudinal study of individual and organisational learning. The Learning Organization, v. 20, n. 3, p. 240-258, 2013. 
CHI, M.T.H; GLASER, R.; FARR, M.J. The nature of expertise. New York: Psychology Press, 2014.

CHURCHILL, Gilbert A.; IACOBUCCI, Dawn. Marketing research: methodological foundations. New York: Dryden Press, 2006.

CLARK, D. Learning domains or Bloom's taxonomy: the three types of learning. Disponivel em: <www. nwlink.com/ donclark/hrd/bloom.html>. Acesso em: 06 jul. 2017.

DARROCH, J. Knowledge management, innovation and firm performance. Journal of knowledge management, v. 9, n. 3, p. 101-115, 2005.

DOROW, P. Compreensão do compartilhamento do conhecimento em atividades intensivas em conhecimento em organizações de diagnóstico por imagem. Tese. (Doutorado em Engenharia e Gestão do Conhecimento) - Universidade Federal de Santa Catarina. Santa Catarina, p. 365. 2017.

DORAN, D. M.; SIDANI, S. Outcomes-Focused Knowledge Translation: A Framework for Knowledge Translation and Patient Outcomes Improvement. Worldviews on Evidence-Based Nursing, v. 4, n. 1, p. 3-13, 2007.

DREYFUS, S.E; DREYFUS, H.L. A five-state model of the mental activities involved in directed skill acquisition. University of California, Berkely, CA: Operation Research Center Report 80-2, 1980.

ERICSSON, K. A. An Introduction to The Cambridge Handbook of expert and Expert Performance: Its Development, Organization, and Content. Cambridge: Cambridge University Press, 2006.

ERICSSON, K. Anders; CHARNESS, Neil. Expert performance: Its structure and acquisition. American psychologist, v. 49, n. 8, p. 725-747, 1994.

ERICSSON, K. Anders; KRAMPE, Ralf T.; TESCH-RÖMER, Clemens. The role of deliberate practice in the acquisition of exper performance. Psychological review, v. 100, n. 3, p. 347-363, 1993.

ERICSSON, K. Anders; LEHMANN, Andreas C. Expert and exceptional performance: Evidence of maximal adaptation to task constraints. Annual review of psychology, v. 47, n. 1, p. 273-305, 1996.

ERICSSON, K. A.; SMITH, J. Toward a general theory of expert: Prospects and limits. Cambridge: Cambridge University Press, 1991.

FITZGERALD, R. Radiological error: analysis, standard setting, targeted instruction and teamworking. European radiology, v. 15, n. 8, p. 1760-1767, 2005.

GIACOMINI, Diogo Schüler; SILVA, Erick Godinho; GRECO, Pablo Juan. Comparação do conhecimento tático declarativo de jogadores de futebol de diferentes categorias e posições. Revista Brasileira de Ciências do Esporte, v. 33, n. 2, p.445-463, 2011.

GUNDERMAN, R.; CHAN, S. Knowledge Sharing in Radiology 1. Radiology, v. 229, n. 2, p. 314-317, 2003.

GUSKEY, T. R. Benjamin S. Bloom's contributions to curriculum, instruction, and school learning. In: ANNUAL MEETING OF THE AMERICAN EDUCATIONAL RESEARCH ASSOCIATION, 3, 2001, Seattle. Proceedings... Seattle: AERA.

HAYES, John; ALLINSON, Christopher W. Cognitive style and the theory and practice of individual and collective learning in organizations. Human relations, v. 51, n. 7, p. 847-871, 1998.

INKINEN, H. T.; KIANTO, A.; VANHALA, M. Knowledge management practices and innovation performance in Finland. Baltic Journal of Management, v. 10, n. 4, p. 432-455, 2015. 
IPÊ, M. Knowledge sharing in organizations: A conceptual framework. Human resource development review, v. 2, n. 4, p. 337-359, 2003.

ISIK, Ö.; MERTENS, W.; VAN DEN BERGH, J. Practices of knowledge intensive process management: quantitative insights. Business Process Management Journal, v. 19, n. 3, p. 515-534, 2013.

JONASSEN, David H.; GRABINGER, R. Scott. Problems and issues in designing hypertext/hypermedia for learning. In D. H. Jonassen \& H. Mandl (Eds.), Designing hypermedia for learning (pp. 3-25). Berlin: Springer.

JORRITSMA, Wiard; CNOSSEN, Fokie; VAN OOIJEN, Peter MA. Adaptive support for user interface customization: a study in radiology. International Journal of Human-Computer Studies, v. 77, p. 1-9, 2015.

KIM, Y. W.; MANSFIELD, L. T. Fool me twice: delayed diagnoses in radiology with emphasis on perpetuated errors. American Journal of Roentgenology, v. 202, n. 3, p. 465-470, 2014.

KRATOCHVIL, Claudia Finger. Letramento e tecnologia: o aprendiz estratégico e crítico na era da informação. In: NASCIMENTO, A. D., HETKOWSKI, T. M., orgs. Educação e contemporaneidade: pesquisas científicas e tecnológicas [online]. Salvador: EDUFBA, 2009, 400 p.

MARCONI, Marina de Andrade; LAKATOS, Eva Maria. Metodologia do trabalho científico: procedimentos básicos, pesquisa bibliográfica, projeto e relatório publicações e trabalhos científicos. São Paulo: Atlas, 2007.

MARZANO, R. J. et al. Dimensions of thinking: A framework for curriculum and instruction. Washington, DC: The Association for Supervision and Curriculum Development, 1988.

MILES, I. Knowledge intensive business services: Prospects and policies. Foresight, v. 7, n. 6, p. 39-63, 2005.

NONAKA, I.; TAKEUCHI, H. The knowledge-creating company: How Japanese companies create the dynamics of innovation. Oxford: Oxford university press, 1995.

PATTI, Giuseppe et al. Point-of-care measurement of clopidogrel responsiveness predicts clinical outcome in patients undergoing percutaneous coronary intervention. Journal of the American College of Cardiology, v. 52, n. 14, p. 1128-1133, 2008.

PETTY, Nicola J.; SCHOLES, Julie; ELLIS, Lorraine. Master's level study: learning transitions towards clinical expertise in physiotherapy. Physiotherapy, v. 97, n. 3, p. 218-225, 2011.

PIZZI, N. J. Information processing in biomedical applications. In: BARGIELA, Andrzej; PEDRYCZ, Witold (Ed.). Human-Centric Information Processing Through Granular Modelling. Maylasia: Springer Berlin Heidelberg, 2009. p. 47-54.

SCHUNK, D. H. Learning theories. New Jersey: Prentice Hall Inc., 1996.

SENGE, P. The fifth discipline: the art and practice of the learning organization. Nova York: DoubledayCurrency, 1990.

SIMON, Herbert A. Bounded rationality and organizational learning. Organization science, v. 2, n. 1, p. 125$134,1991$.

SMITH, P. L.; RAGAN, T.J. Instructional design. New York: Wiley, 1999.

STERNBERG, Robert J. Psicologia Cognitiva. Porto Alegre: Artmed Editora, 2000.

STERNBERG, Robert J.; GRIGORENKO, Elena L. (Ed.). The psychology of abilities, competencies, and expertise. Cambridge: Cambridge University Press, 2003. 
TARDIF, J., Pour un Enseignement Stratégique: L'apport de la Psychologie Cognitive. Montréal: Editions Logiques, 1997.

TRIVIÑOS, Augusto Nibaldo Silva. Introdução à pesquisa em ciências sociais: a pesquisa qualitativa em educação. São Paulo: Atlas, 1987.

VAN DE WIEL, Margaretha WJ; SZEGEDI, Kim HP; WEGGEMAN, Mathieu CDP. Professional learning: Deliberate attempts at developing expertise. In: Professional learning: Gaps and transitions on the way from novice to expert. Springer, Dordrecht, 2004. p. 181-206.

WILLIAMSON, Kenneth B. et al. Learning theory in radiology education. Radiology, v. 233, n. 1, p. 15-18, 2004. 